\title{
Simultaneous application of transcranial magnetic stimulation and low-frequency electrostatic field as treatment of carbohydrate and lipid disorders in patients with metabolic syndrome
}

Andrey A. Benkov (1), Sergey N. Nagornev (2), Valery K. Frolkov (2), Valentine F. Reps (3), Dmitriy A. Edelev (4), Anatoly D. Fesyun (5), Maxim Yu. Yakovlev (5,6), Natalia Fedorovna Tumanova (5), Natalia P. Sanina (7), Maria Chiara Maccarone (8), Stefano Masiero $(8,9)$

(1) Limited Liability Company "Med TeKo", Moscow, Russia; (2) Federal State Budgetary Institution "Center for Strategic Planning and Management of Biomedical Health Risks" of the Federal Biomedical Agency of the Russian Federation, Moscow, Russia; (3) FGBU "Pyatigorsk State Research Institute of Balneology of the Federal Medical and Biological Agency of the Russian Federation, Moscow, Russia; (4) Federal State Budgetary Educational Institution of Higher Education "Lomonosov Moscow State University", Moscow, Russia; (5) FGBU "National Medical Research Center for Rehabilitation and Balneology" of the Ministry of Health of the Russian Federation, Moscow, Russia; (6) Federal State Autonomous Educational Institution of Higher Education I.M. Sechenov First Moscow State Medical University (Sechenov University), Ministry of Health of Russia, Moscow, Russia. (7) Moscow regional research clinical institute named after M.F. Vladimirskiy; (8) Physical Medicine and Rehabilitation School, University of Padova, Padua, Italy; (9) Rehabilitation Unit, Department of Neuroscience, University of Padova, Padua, Italy.

This article is distributed under the terms of the Creative Commons Attribution Noncommercial License (CC BY-NC 4.0) which permits any noncommercial use, distribution, and reproduction in any medium, provided the original author(s) and source are credited.

\begin{abstract}
The metabolic syndrome, which covers a wide variety of pathological concerns, is rapidly becoming a global pandemic. This syndrome is difficult to treat pharmacologically. Physiotherapy techniques, which have both local and systemic effects, can be employed as a suitable substitute. The purpose of this study was to investigate the therapeutic effects of a program of simultaneous physiotherapy that included migrant transcranial magnetic stimulation (TMS) and the exposure to an alternating low-frequency electrostatic field (LFEF) in the treatment of metabolic syndrome patients. Ninety patients were randomly assigned to three study groups. While continuing the usual drug therapy the first group (30 patients) received LFEF intervention, the second group (30 patients) received TMS, and the third group (30 patients) underwent the simultaneous use of these non-invasive techniques (LFEF + TMS). All treatments involved 10 sessions with daily frequency. In all the patients before and after treatment body weight, blood pressure parameters, levels of insulin, cortisol, glucose, total cholesterol, high density lipoproteins, malondialdehyde, and Schiff bases, the activity of the antioxidant enzymes catalase and of the superoxide dismutase were studied. The changes in the outcomes assessed revealed a different reaction to therapy with LFEF or TMS, as well as a greater benefit when both treatments were used at the same time. A simultaneous LFEF and TMS intervention seems a promising resource for the treatment of the metabolic syndrome, particularly of the lipid and carbohydrate metabolism disorders. However, further studies are needed to confirm these findings and investigate the underlying mechanisms.
\end{abstract}

Key Words: Low-frequency electrostatic field; transcranial magnetic stimulation; obesity; cortisol; insulin resistance.

Eur J Transl Myol 32 (1): 10351, 2022 doi: 10.4081/ejtm.2022.10351

The metabolic syndrome, which covers a wide variety of pathological concerns, the most prominent of which is a dysfunction of carbohydrate and lipid metabolism in the context of arterial hypertension, is rapidly becoming a 
global pandemic, accelerating the progression of other diseases and increasing the frequency of cardiovascular accidents and type 2 diabetes. ${ }^{1-4}$

Metabolic syndrome is defined as the occurrence of three abnormal results out of five components: elevated waist circumference, elevated triglycerides, reduced highdensity lipoprotein cholesterol, elevated blood pressure, and elevated fasting plasma glucose. ${ }^{4}$

Up to $50 \%$ of the population over 60 may be affected by metabolic syndrome with important clinical repercussions. $^{5}$

Because of the range of adverse reactions and the existence of side effects in most medications, pharmacological therapy for this syndrome is challenging. ${ }^{6,7}$ Physiotherapy approaches, which can have both local and systemic impact, can be used as an appropriate alternative. .,9 $^{2}$

However, the feasibility of applying non-invasive instrumental physical therapies to these patients is still poorly investigated. This is even more true when considering instrumental physical therapies combined at the same time (one-step or simultaneous program), whose benefits have been demonstrated by recent studies. ${ }^{10,11}$ Instrumental physical therapies simultaneously used have a greater synergy effect on their physiological and therapeutic targets. Moreover, habituation to the combined regimen occurs in a significantly lower percentage of cases. ${ }^{12}$ Due to the active participation of the neurohumoral regulation system, the combined employment of physical factors is accompanied by a broad nonspecific reaction of the body. In this perspective, the action of instrumental physical therapies can be considered as an irritant effect that causes the human body to undergo rapid adaptation processes, resulting in the creation of a high resistance state. The nonspecific stress reaction has a prominent role in the organization of the adaptation processes. ${ }^{13-15}$

In this regard, two non-invasive techniques as the transcranial magnetic stimulation with a migrant transcranial magnetic stimulation (TMS) field and the exposure to an alternating low-frequency electrostatic field (LFEF) are of particular interest. Neuromodulatory approaches represent a promising tool for targeting specific brain structures implicated in the pathophysiology of obesity. ${ }^{16}$ The studies of A. V. Kulish proved that TMS effectively acts on the regulatory mechanisms in the central nervous system, ${ }^{17}$ including the hormonal mechanisms involved in carbohydrate and lipid metabolism, while LFEF stimulates antioxidant and microcirculatory processes. ${ }^{18}$ In the metabolic syndrome, changes in these processes largely explain the multiplicity of pathological manifestations, therefore the possibility of a simultaneous correction is of great clinical interest.

However, to our knowledge there are currently no studies that have investigated how these two noninvasive techniques can act simultaneously on patients with metabolic syndrome.

\section{Materials and Methods}

\section{Study design}

We conducted a randomized trial, dividing enrolled patients randomly into three treatment groups.

\section{Participants}

Subjects of both sexes over the age of 18 who have a diagnosis of metabolic syndrome were included in the study. Enrolled subjects must be able to provide informed consent. Heart failure, respiratory failure, impaired cognitive functions (mini mental status examination < 24), epilepsy, pregnancy, oncological or psychiatric comorbidities, and inability to properly comprehend and sign informed consent were the exclusion criteria.

\section{Intervention and procedure}

The study involved 90 patients suffering from metabolic syndrome. Patients were randomly assigned to three study groups. The first group (30 patients) received LFEF procedures, the second group (30 patients) received TMS, and the third group (30 patients) underwent the simultaneous use of these non-invasive techniques (LFEF + TMS). All patients continued with the usual therapy previously prescribed (diet, antihypertensive drugs, physiotherapy exercises). Exposure to an alternating LFEF was carried out using the multifunctional therapeutic system "Khivamat-200". The procedures were performed using the manual option and carried out on the neck area with a frequency of $100 \mathrm{~Hz}$, on a daily basis, with an intensity of $50 \%$, a duration of 10-12 minutes, for a total of 10 sessions. TMS was practiced with the "Amo-Atos" device with "Headgear", two semi-cylindrical emitters bitemporally located, alternating magnetic field. The therapy was carried out in a sitting position, starting with a frequency of $1 \mathrm{~Hz}$, a duration of $7 \mathrm{~min}$, and a field strength of 10-30 mT. Then the frequency and the duration of the procedure were gradually increased to $10 \mathrm{~Hz}$ and $12 \mathrm{~min}$, respectively, which made it possible to achieve a state of adaptation to this non-invasive technique and to exclude individual intolerance. The magnitude of the magnetic induction of 10-30 mT provides a sufficient penetration depth of the magnetic field when exposed to the diencephalic structures of the brain. Magnetotherapy program included 10 daily sessions. The research procedures conformed to the Declaration of Helsinki.

\section{Outcome Measures}

In all the patients before and after treatment body weight, blood pressure parameters, levels of insulin, cortisol, glucose, total cholesterol, high density lipoproteins, malondialdehyde, Schiff bases, and the activity of the antioxidant enzymes catalase and of the superoxide dismutase were studied. Hormonal parameters were determined by enzyme immunoassay, glucose and lipids levels were measured with a biochemical analyzer, and the pro- and anti-oxidant molecules through methods explained in previous papers by V. B. Gavrilov et al. ${ }^{19}$ 
and M. A. Korolyuk et al. ${ }^{20}$ Calculation methods were used to determine the index of insulin resistance, ${ }^{21}$ and the coefficient of atherogenicity. ${ }^{22}$ A set of 18 healthy adults was used to create the reference values, and they remained unchanged throughout the study.

\section{Statistical Method}

Statistical processing of the obtained results was carried out using the Statistica 12.6 software (StatSoft, USA), using parametric methods for assessing the reliability of statistical differences, as well as the correlation analysis. The level of statistical significance was set at $95 \%$ and the statistical tests used for analysis included: Spearman's rank correlation, Mann-Whitney Test for categorical/factorial variables, and Student's t-test for quantitative variables

\section{Results}

The main results obtained in the comparative study of indicators in patients with metabolic syndrome and a group of healthy subjects are presented in Table 1 .

The study involved 90 patients (49 women and 41 men aged 44.6 \pm 0.31 years) suffering from metabolic syndrome with an initial state characterized by increased Body Mass Index (BMI), increased level of blood pressure, severe dyslipidemia, significant increase in insulin production, and decreased sensitivity to insulin (BMI 30.8 \pm 0.14 , blood pressure above 140/90 mm Hg, insulin resistance index 5.93 \pm 0.09 , atherogenic

Table 1. Demographic and clinical characteristics of patients with metabolic syndrome and healthy volunteers.

\begin{tabular}{|c|c|c|}
\hline Indicators & $\begin{array}{l}\text { Patients with metabolic } \\
\text { syndrome }\end{array}$ & $\begin{array}{c}\text { Normal values } \\
\text { (healthy volunteers) }\end{array}$ \\
\hline BMI & $30,9 \pm 0,14^{* *}$ & $24,8 \pm 0,17$ \\
\hline Systole blood pressure, mmHg & $144 \pm 1,06 * *$ & $124 \pm 2,7$ \\
\hline Diastole Blood pressure, mmHg & $92 \pm 0,79 * *$ & $83 \pm 1,9$ \\
\hline Total cholesterol, mmol/L & $6,26 \pm 0,07^{* *}$ & $3,74 \pm 0,15$ \\
\hline High density lipoproteins, mmol/L & $1,02 \pm 0,04 *$ & $1,19 \pm 0,07$ \\
\hline Atherogenic coefficient & $4,96 \pm 0,08 * *$ & $2,14 \pm 0,07$ \\
\hline Insulin, $\mu \mathrm{U} / \mathrm{mL}$ & $23,9 \pm 0,22 * *$ & $11,5 \pm 0,38$ \\
\hline Glucose, mol/L & $5,65 \pm 0,13^{* *}$ & $4,71 \pm 0,16$ \\
\hline Insulin resistance index (HOMA) & $6,00 \pm 0,18^{* *}$ & $2,41 \pm 0,09$ \\
\hline Cortisol, nmol/L & $421 \pm 9,3 * *$ & $302 \pm 15,6$ \\
\hline Malondialdehyde, nmol/mL & $8,08 \pm 0,19 * *$ & $5,77 \pm 0,26$ \\
\hline Schiff bases, rel.units/mL & $6,27 \pm 0,13^{* *}$ & $4,90 \pm 0,19$ \\
\hline Catalase, active units/g Hb & $881 \pm 16,9 * *$ & $1073 \pm 25,8$ \\
\hline Superoxide dismutase, active units/g Hb & $104 \pm 2,5^{* *}$ & $126 \pm 4,1$ \\
\hline
\end{tabular}

Note: Superscript indices show the significance in comparison with normal values $(*-p<0.05 ; * *-p<0.01)$. 
Table 2. Mean values of outcomes and standard deviations before and after interventions in the different groups.

\begin{tabular}{|c|c|c|c|}
\hline Indicators & Group 1 (LFEF) & Group 2 (TMS) & Group 3 (LFEF+TMS) \\
\hline BMI & $\begin{array}{l}30,5 \pm 0,24 \\
30,1 \pm 0,22\end{array}$ & $\begin{array}{l}31,0 \pm 0,27 \\
29,9 \pm 0,20 *\end{array}$ & $\begin{array}{l}31,2 \pm 0,29 \\
29,6 \pm 0,34 *\end{array}$ \\
\hline Systole blood pressure, mmHg & $\begin{array}{l}143 \pm 2,16 \\
138 \pm 2,03\end{array}$ & $\begin{array}{l}145 \pm 2,21 \\
135 \pm 1,84 *\end{array}$ & $\begin{array}{l}144 \pm 2,23 \\
130 \pm 2,70^{*}\end{array}$ \\
\hline Diastole Blood pressure, mmHg & $\begin{array}{l}90 \pm 1,35 \\
88 \pm 1,12\end{array}$ & $\begin{array}{l}92 \pm 1,44 \\
84 \pm 1,08 *\end{array}$ & $\begin{array}{l}92 \pm 1,38 \\
83 \pm 1,51^{*}\end{array}$ \\
\hline Glucose, mmol/L & $\begin{array}{l}5,64 \pm 0,17 \\
5,39 \pm 0,13 \\
\end{array}$ & $\begin{array}{l}5,75 \pm 0,18 \\
5,52 \pm 0,15 \\
\end{array}$ & $\begin{array}{l}5,57 \pm 0,15 \\
5,06 \pm 0,21 \\
\end{array}$ \\
\hline Total cholesterol, mmol/L & $\begin{array}{l}6,29 \pm 0,15 \\
5,86 \pm 0,12\end{array}$ & $\begin{array}{l}6,32 \pm 0,16 \\
5,94 \pm 0,14\end{array}$ & $\begin{array}{l}6,07 \pm 0,15 \\
5,12 \pm 0,24 *\end{array}$ \\
\hline High density liproteins, mmol/L & $\begin{array}{l}1,03 \pm 0,05 \\
1,09 \pm 0,06\end{array}$ & $\begin{array}{l}1,04 \pm 0,05 \\
1,06 \pm 0,07\end{array}$ & $\begin{array}{l}0,97 \pm 0,04 \\
1,10 \pm 0,08\end{array}$ \\
\hline Atherogenic coefficient & $\begin{array}{l}5,11 \pm 0,13 \\
4,38 \pm 0,10^{*}\end{array}$ & $\begin{array}{l}5,08 \pm 0,14 \\
4,60 \pm 0,12 *\end{array}$ & $\begin{array}{l}5,26 \pm 0,15 \\
3,65 \pm 0,15 *\end{array}$ \\
\hline Insulin, $\mu \mathrm{U} / \mathrm{mL}$ & $\begin{array}{l}23,6 \pm 0,39 \\
21,2 \pm 0,34\end{array}$ & $\begin{array}{l}24,2 \pm 0,38 \\
20,3 \pm 0,31 *\end{array}$ & $\begin{array}{l}23,8 \pm 0,37 \\
17,4 \pm 0,42 *\end{array}$ \\
\hline Insulin resistance index & $\begin{array}{l}5,92 \pm 0,17 \\
5,08 \pm 0,14^{*}\end{array}$ & $\begin{array}{l}6,18 \pm 0,20 \\
4,98 \pm 0,13 *\end{array}$ & $\begin{array}{l}5,89 \pm 0,15 \\
3,91 \pm 0,19 *\end{array}$ \\
\hline Cortisol, nmol/L & $\begin{array}{l}409 \pm 27,50 \\
381 \pm 21,30 \\
\end{array}$ & $\begin{array}{l}420 \pm 28,30 \\
468 \pm 30,70 *\end{array}$ & $\begin{array}{l}433 \pm 30,6 \\
498 \pm 38,4^{*}\end{array}$ \\
\hline Malonic dialdehyde, nmol/mL & $\begin{array}{l}8,05 \pm 0,30 \\
7,12 \pm 0,25^{*}\end{array}$ & $\begin{array}{l}8,17 \pm 0,31 \\
7,55 \pm 0,27\end{array}$ & $\begin{array}{l}7,91 \pm 0,27 \\
6,25 \pm 0,36 *\end{array}$ \\
\hline Schiff bases, rel.units/mL & $\begin{array}{l}6,12 \pm 0,23 \\
4,94 \pm 0,12 *\end{array}$ & $\begin{array}{l}6,04 \pm 0,20 \\
5,63 \pm 0,16\end{array}$ & $\begin{array}{l}6,40 \pm 0,24 \\
5,03 \pm 0,24^{*}\end{array}$ \\
\hline Catalase, active units/g Hb & $\begin{array}{l}897 \pm 23,4 \\
1015 \pm 28,0 *\end{array}$ & $\begin{array}{l}915 \pm 25,2 \\
980 \pm 27,1 \\
\end{array}$ & $\begin{array}{l}859 \pm 21,90 \\
1085 \pm 35,0 *\end{array}$ \\
\hline $\begin{array}{l}\text { Superoxide dismutase, active } \\
\text { units/g Hb }\end{array}$ & $\begin{array}{l}102 \pm 3,51 \\
121 \pm 4,24 *\end{array}$ & $\begin{array}{l}97 \pm 3,14 \\
106 \pm 3,70\end{array}$ & $\begin{array}{l}110 \pm 3,82 \\
129 \pm 4,97 *\end{array}$ \\
\hline
\end{tabular}

Note: In each cell of the table, the upper values are before the treatment, the lower ones are after the treatment. An asterisk marks statistically significant changes of the indicators after treatment $\left.*^{*}-p<0.05\right)$.

coefficient 5.19 \pm 0.07 ) (Table 1). The insulin resistance index seemed to correlate with several indicators of metabolic syndrome. Spearman's rank correlation coefficient between insulin resistance index and others indicators was $\rho=+0.62(\mathrm{p}<0.001)$ for BMI, $\rho=+0.43$ $(\mathrm{p}<0.01)$ for blood pressure, $\rho=+0.58(\mathrm{p}<0.001)$ for atherogenic coefficient, $\rho=+0.32(p<0.05)$ for cortisol level, $\rho=+0.39(\mathrm{p}<0.01)$ for malondialdehyde level, $\rho=$ $+0.30(\mathrm{p}<0.05)$ for Schiff bases level, $\rho=-0.52(\mathrm{p}$ $<0.001)$ for catalase level, and $\rho=-0.46(\mathrm{p}<0.01)$ for superoxide dismutase level. Analysis of change in indicators after treatment with noninvasive physical therapies showed a different response to treatment with LFEF or TMS and greater benefit with the simultaneous treatments. LFEF seemed to manifest its biological effect mainly in lipid peroxidation and glucocorticoid activities. In particular, the pro-oxidant action seemed to be significantly inhibited: the level of malondialdehyde decreased by $11.5 \%$ and the level of Schiff bases by
$19.3 \%$, while there was an increase in the activity of antioxidant enzymes (catalase and superoxide dismutase increased of $13.2 \%$ and $18.6 \%$ respectively). At the same time, there was a significant decrease in the atherogenic coefficient and in the insulin resistance index, by $14.3 \%$ and $14.2 \%$ respectively. No significant changes in BMI, blood pressure indicators, lipids and glucose concentration in the blood were found, while a slight reduction in the production of cortisol was recorded (Table 2).

After TMS, more pronounced changes in the systemic manifestations of the metabolic syndrome were noted: a decrease in BMI of 3.6\% and in blood pressure of 7-9\%, and a major decrease in insulin resistance index of $19.4 \%$ were found. No significant changes in the system of lipid peroxidation were observed. Cortisol secretion did not decrease, in contrast, it increased slightly (11.4\%), as evidenced by the application of the Student's t- test for comparable groups. 


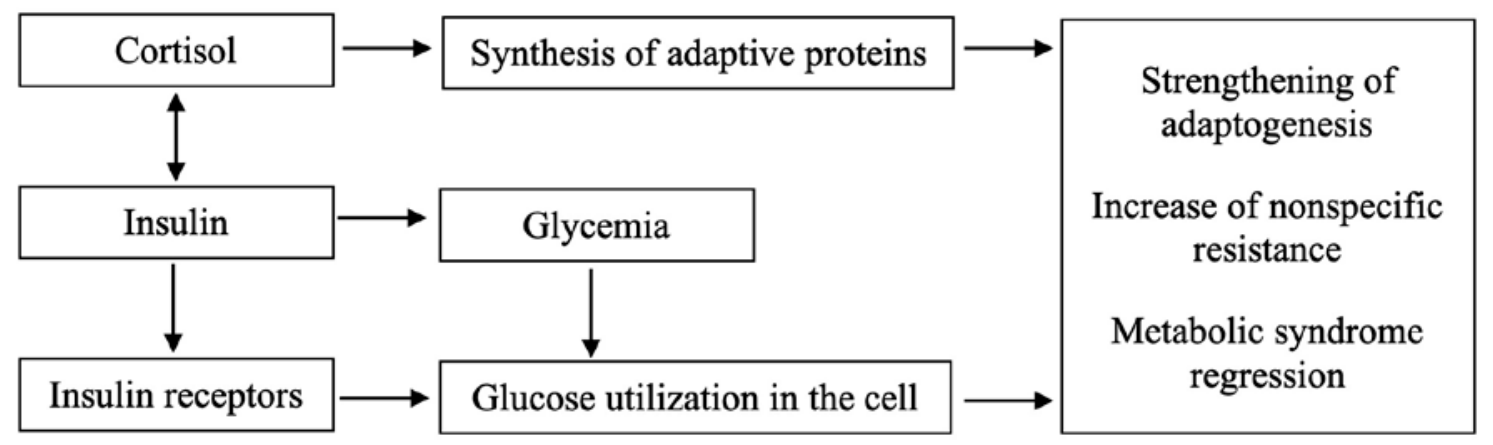

Fig 1. Implementation of the metabolic effects of insulin and cortisol.

With the simultaneous application of LFEF and TMS significant changes were obtained in almost all indicators. According to the variation in BMI, blood pressure, activity of pro- and antioxidant reactions, the synergic effects of LFEF and TMS were noted. Moreover, the decrease in the atherogenic coefficient and in the insulin resistance index (30.7\% and 33.6\% respectively) was more pronounced than with monoexposure. With the simultaneous treatment, cortisol level increased more (15.0\%) than with mono-exposure intervention (Table 2).

\section{Discussion}

After the treatment with noninvasive physical therapies, the changes recorded in the outcomes evaluated demonstrated different reactions to LFEF or TMS, as well as a greater benefit when both treatments were utilized at the same time. After LFEF intervention, patients underwent a reduction in the pro-oxidant activity and an increase in the activity of antioxidant enzymes, a significant decrease in the atherogenic coefficient and in the insulin resistance index, and a slight reduction in the production of cortisol. On the other hand, after TMS, pronounced changes in the systemic manifestations of the metabolic syndrome were noted including a decrease in $\mathrm{BMI}$, in blood pressure, and in insulin resistance index. Moreover, cortisol secretion increased in this treatment group. With the simultaneous application of LFEF and TMS significant changes were obtained in almost all indicators, reconfirming the synergistic effect of these treatments. Furthermore, the decrease in the atherogenic coefficient and in the insulin resistance index and the increase in cortisol level were more pronounced. The correlation between the insulin resistance index and the evaluated indicators matches with recent notions concerning the polymodality of disease pathophysiology, which suggest the development of a premorbid background in several critical systems., ${ }^{5,23,24}$ Our findings corroborate the generally held belief that tissue resistance to insulin, the body's main hypoglycemic agent, is a crucial mechanism driving the development of metabolic syndrome. ${ }^{5}$ It is worth noting that glycemic homeostasis is always disrupted first, whereas detrimental changes in lipid metabolism develop over time and become noticeable only after a few years. ${ }^{5}$

Insulin resistance is poorly susceptible to pharmaceutical repair. Insulin receptors can be classed as channelforming receptors in terms of systems biology. Because a cascade of reactions is triggered when the receptor and the ligand (insulin) interact, the GLUT4 transport protein is activated. This carrier protein forms a transmembrane channel for glucose. Bobrovnitsky et al. ${ }^{25}$ showed that one of the possible mechanisms for the restoration of insulin-receptor interaction may be the activation of the processes of adaptogenesis. In the context of physiotherapeutic therapies aimed at restoring impaired self-regulation, two possible strategies for increasing insulin receptor sensitivity can be distinguished:

- antioxidant mechanism, due to the peroxide modifications of the receptor and its contact sites for insulin interaction are eliminated;

- neurohumoral mechanism promoting the receptor modification. This mechanism also increases the availability and affinity of both receptor sites for the ligand.

At the same time, it can be noted that the compensatory enhancement of energy metabolism during the formation of adaptive processes is a secondary reaction to the previous stressor effect, which is realized also with the participation of stress hormones of the hypothalamicpituitary-adrenal system - ACTH and cortisol. As a result, stimuli with a moderate stress-initiating potential should be employed to activate insulin regulation of glucose and lipid metabolism, which are the primary substrates for ATP production. Hyperglycemia is usually an advisable reaction since it increases the mobilization of energy resources, however glycogen stores are usually short-lived. Therefore, with long-term processes, the intensification of energy homeostasis is provided due to gluconeogenesis, whose main inducer is cortisol. This concern is interesting from a variety of perspectives. It is known that insulin, cortisol and glucose are most actively 
involved in the formation of adaptive responses at all stages of adaptogenesis - from the activation of stress mechanisms to the increased activity of stress-limiting systems (endogenous opiates, antioxidant enzymes). In general, the algorithm for the implementation of the metabolic effects of insulin and cortisol is shown in Figure 1.

Current therapeutic options for metabolic syndrome are limited to individual treatments for hypertension, hyperglycemia, and hypertriglyceridemia, as well as dietary control measures and regular exercise, including water-based training. ${ }^{24,26,27}$ It is reasonable to believe that the development of an innovative therapeutic and preventative algorithm aimed at influencing metabolic processes and enhancing body's resistance to pathogenic stimuli should not only consider, but also actively exploit natural reactions. An example of the validity of this postulate can be derived from the thousand-year experience of traditional medicine with powerful herbal adaptogens (phytopreparations of ginseng, leuzea, polyascis, etc.) that, when first used, have a pronounced stimulating effect on the pituitary-adrenal system, ${ }^{28}$ provoking endocrine stress reactions. Moreover, they can act on major antioxidant enzymes, reducing Reactive Oxygen Species generated during oxidative stress and inhibiting apoptosis. ${ }^{29}$ These facts support one of the main theses of restorative medicine: improving health and/or restoring functional reserves can be achieved if sufficient reserves exist in the systems firstly involved and in which a stress-initiating signal is created, while insufficient reserves may be the cause of an excessive stress response. The influence on the body's reserves, the absence of side effects attributable to drug chemical properties, and the capacity to assess and anticipate the therapeutic and prophylactic effect based on the amplitude of the first stress reaction are the main advantages of this technique.

However, even if this hypothesis is interesting, studies on the topic are very poor. Also human body's response to the instrumental physical factors includes a certain set of nonspecific reactions, among which adaptation reactions, stress-initiating and stress-limiting processes are undoubtedly present. Through the simultaneous use of TMS and LFEF in patients with metabolic syndrome, the impairment of carbohydrates and lipids metabolism, that creates a background for cardiovascular diseases and diabetes mellitus, can be compensated activating physiological reactions.

In particular, our findings seem to suggest a restorative action on the main metabolic hormones (insulin and cortisol), which could also lead to an improvement in the functioning of the entire cardiovascular system. Indeed, the endocrine reaction aroused by the simultaneous application of these physical instrumental factors determines, according to the principle of cross adaptation, changes in the metabolism not only in a single organ, but also in the whole body. The changes in metabolism throughout the body can represent not only a valid treatment, but can represent also a valid prevention strategy, involving organs and systems not yet affected by the disease. The increase in plasma cortisol levels was shown in our study after the TMS and the simultaneous treatments. The benefits of a rise in cortisol values could be related to the activation of insulin regulation of glucose and lipid metabolism, as mentioned above. However, we did not analyze a control group performing placebo treatment because it would have been difficult to discriminate the placebo effect from a possible minimal therapeutic effect (application of non-invasive methods working at reduced dosages).

Clinical studies have shown that cortisol levels can increase following stimulations such as noise and heat. ${ }^{30}$ Therefore, it cannot be excluded that the results obtained derive not only from the use of specific physical instrumental factors but also from the nonspecific effect of therapies.

Our study presents two main limitations. Firstly, the small sample size may have affected the results obtained. Secondly, the sample was not compared with a placebo control group. As a result, the current study's findings are encouraging but not conclusive.

In conclusion, the simultaneous use of TMS and LFEF seems promising in the treatment of metabolic syndrome, particularly because of the beneficial effects obtained on lipid and carbohydrate metabolism. However, further studies are needed to better understand the mechanisms behind these results, allowing in the future to develop innovative protocols for the treatment of these patients.

\section{List of acronyms}

BMI - body mass index

TMS -transcranial magnetic stimulation

LFEF - low-frequency electrostatic field

\section{Contributions of Authors}

SNN and VKF: Study concept and design; AAB, NFT, MYuY: Collection of materials; VKF and MYuY: Statistical processing; SNN, VFR, DAE, ADF: Analysis of the data obtained; AAB, NPS,MCC: Text writing; SNN, MCC, SM: Editing. All contributors approved the manuscript and agreed with study publication.

\section{Acknowledgments}

The authors hereby thank the patients and volunteers, who aided in conducting the present study.

\section{Funding}

The authors received no specific funding for this work.

\section{Conflict of Interest}

The authors declare no conflict of interests.

\section{Ethical Publication Statement}

We confirm that we have read the Journal's position on issues involved in ethical publication and affirm that this report is consistent with those guidelines. 


\section{Corresponding Author}

Maria Chiara Maccarone, Physical Medicine and Rehabilitation School, University of Padova, Via Giustiniani 3, 35128 Padova, Italy.

ORCID iD: ORCID iD: 0000-0003-2793-1334

Email: mariachiara.maccarone@studenti.unipd.it

E-mails and ORCID iD of co-authors

Andrey A. Benkov: a.benkov@medteco.ru

ORCID iD: 0000-0003-4074-7208

Sergey N. Nagornev: drnag@mail.ru

ORCID iD: 0000-0002-1190-1440

Valery K. Frolkov: fvk49@mail.ru

ORCID iD: 0000-0002-1277-5183

Valentine F. Reps: v.reps@mail.ru

ORCID iD: 0000-0003-4878-6797

Dmitriy A. Edelev: edelevda@gmail.com

ORCID iD: 0000-0002-5863-2284

Anatoly D. Fesyun: fesyunad@nmicrk.ru

ORCID iD: 0000-0003-3097-8889

Maxim Yu.Yakovlev: masdat@mail.ru

ORCID iD: /0000-0002-5260-8304

Natalia Fedorovna Tumanova: ntumanova@yandex.ru

ORCID iD: 0000-0002-7021-857X

Natalia P. Sanina: nataliasanina2@yandex.ru

ORCID iD: 0000-0001-8597-1962

Stefano Masiero: stef.masiero@unipd.it

ORCID iD: 0000-0002-0361-4898

\section{References}

1. Uspensky $\mathrm{Yu} \mathrm{P}$, Fominykh $\mathrm{Yu}$ A, Ivanov SV, Niyazov RM. Metabolic syndrome as a problem of topical medicine and the future of mankind. Medicine: theory and practice. 2018;3(1):95-99.

2. Sokolov DA The risk of developing cardiovascular diseases in people with metabolic syndrome. Bulletin of the Russian Military Medical Academy. 2019;3 (1): 15-17.

3. Fedash VV. Association of insulin resistance with hypertension and the risk of cardiovascular diseases. Science Time (Moldova). 2020;11(83): S.56-62.

4. Nilsson PM, Tuomilehto J, Rydén L. The metabolic syndrome - What is it and how should it be managed?. Eur J Prev Cardiol. 2019;26 (2_suppl):33-46. doi:10.1177/2047487319886404.

5. McCracken E, Monaghan M, Sreenivasan S. Pathophysiology of the metabolic syndrome. Clin Dermatol. 2018;36(1):14-20. doi:10.1016/j.clinder matol.2017.09.004

6. Kulchitskaya DB, Turova EA, Nesterova EV. Application of non-drug methods of treatment in patients with metabolic syndrome. Questions of balneology, physiotherapy and medical physical culture. 2020;97(6-2:63-64.

7. Khadartsev AA, Logatkina AV, Terekhov IV, Bondar SS. Dynamics of manifestations of metabolic syndrome in patients with arterial hypertension against the background of the combined use of low-intensity microwave therapy. Arterial hypertension. 2018;24(2):206-216.

8. Ponomarenko GN. Innovative technologies of physiotherapy. Topical issues of physiotherapy: Selected lectures. 2010; pag. 112-135.

9. Fedotchenko AA, Fedotchenko AA. Electrophysiological and neurohumoral mechanisms of physiotherapy. Acta Biomedica Scientifica. 2017;2(4):115-118.

10. Ulashchik VS. Combined physiotherapy: general information, interaction of physical factors. Problems of balneology, physiotherapy and medical physical culture. 2016;6:4-11.

11. Orekhova EM, Konchugova TV, Kul'chitskaya DB, Korchazhkina NB, Egorova LA, Chuich NG. Modern approaches to the use of neurotropic physical therapy in arterial hypertension. Vopr Kurortol Fizioter Lech Fiz Kult. 2016 MayJun;93(3):53-55. Russian. doi: 10.17116/kurort 2016353-55.

12. Xiao LJ, Tao R. Physical Therapy. Adv Exp Med Biol. 2017;1010:247-260. doi:10.1007/978-98110-5562-1_12.

13. Yakovlev M Yu, Tumanova-Ponomareva NF, Fesyun AD, Ismailova LA, Ambrazhuk II The effectiveness of the school of health in a sanatorium for patients with acute coronary syndrome. Russian Journal of Rehabilitation Medicine. 2020;3:78-89.

14. Saltykova MM, Shopina OV, Balakaeva AV, Bobrovnitskiy IP Air pollution as a factor in the increased mortality of the population. Russian Journal of Rehabilitation Medicine. 2020;4:4-16.

15. Bobrovnitskiy IP, Nagornev SN, Khudov VV, Yakovlev My. The main directions of improving the activities of health centers in the Arctic zone of the Russian Federation. Russian Journal of Rehabilitation Medicine. 2020;3:35-55.

16. Göbel CH, Tronnier VM, Münte TF. Brain stimulation in obesity. Int $\mathrm{J}$ Obes (Lond). 2017;41(12):1721-1727. doi:10.1038/ijo.2017.150

17. Kulish AV. A systematic approach to the use of transcranial magnetic effects in the rehabilitation of patients with chronic non-infectious diseases. 2017; p. 48

18. Kulikov AG, Yarustovskaya OV, Kuzovleva EV. Application of low-frequency electrostatic field in clinical practice. Russian Journal of Physiotherapy, Balneology and Rehabilitation. 2019;18(3):195209. doi: 10.17816/1681-3456-2019-18-3-195-209.

19. Gavrilov VB, Gavrilova AR, Mazhul LM. Analysis of methods for the determination of LPO products in serum by the test with TBC. Questions of medical chemistry. 1987;1:118-122.

20. Korolyuk MA, Ivanova LI, Mayorova IG, Tokarev VE. Method for determining the activity of catalase. Clinical laboratory diagnostics. 1988;1:16-19. 
21. Matthews DR, Hosker JP, Rudenski AS, Naylor BA, Treacher DF, Turner RC. Homeostasis model assessment: insulin resistance and beta-cell function from fasting plasma glucose and insulin concentrations in man. Diabetologia. 1985 Jul;28(7):412-9. doi: 10.1007/BF00280883.

22. Klimov AN, Parfenova NS, Golikov IuP One century of the cholesterol model of atherosclerosis /Biomed Khim 2012;58(1):5-11. doi: 10.18097/ pbmc20125801005.

23. Mikhailenko LV, Nagornev SN, Bobrovnitsky IP, Frolkov VK. Phytotherapy of arterial hypertension in combination with obesity in sanatorium-resort conditions. Questions of balneology, physiotherapy and medical physical culture. 2009;5:13-17.

24. Mikhailenko LV, Bobrovnitsky IP, Nagornev SN, Frolkov VK. Predictor significance of the initial morphological and functional indicators in assessing the effectiveness of the rehabilitation correction of arterial hypertension. Questions of balneology, physiotherapy and medical physical culture. 2010;2:35-38.

25. Frolkov V K, Edelev D A Mineral waters effects on metabolic syndrome (a clinico-experimental trial) Vopr Kurortol Fizioter Lech Fiz Kult. 2006;(3):26-8.

26. Masiero S, Vittadini F, Ferroni C, Bosco A, Serra R, Frigo AC, Frizziero A. The role of thermal balneotherapy in the treatment of obese patient with knee osteoarthritis. Int J Biometeorol. 2018
Feb;62(2):243-252. doi: 10.1007/s00484-0171445-7. Epub 2017 Sep 22.

27. Masiero S, Maccarone MC, Agostini F. Health resort medicine can be a suitable setting to recover disabilities in patients tested negative for COVID19 discharged from hospital? A challenge for the future. Int J Biometeorol. 2020;64(10):1807-1809. doi:10.1007/s00484-020-01947-4

28. Filaretov AA, Vassilevskaya LV. Electrical activity of hypothalamus during activation of pituitaryadrenocortical system Brain Res. 1978 Nov 3;156(1):67-74. doi: 10.1016/0006-8993(78) 90079-3.

29. Wei W, Li X, Hao J, Zhang R, Guo J, Zong Y, Lu Y, Qu S, Tian J. Proteomic analysis of functional dyspepsia in stressed rats treated with traditional Chinese medicine "Wei Kangning". J Gastroenterol Hepatol. 2011 Sep;26(9):1425-33. doi: 10.1111/j. 1440-1746.2010.06566.x.

30. Jafari MJ, Khosrowabadi R, Khodakarim S, Khodagholi F, Mohammadian F. The effects of combined exposure to noise and heat on human salivary cortisol and blood pressure. Int J Occup Saf Ergon. 2021 Sep;27(3):831-839. doi: 10.1080/ 10803548.2019.1659578. Epub 2019 Oct 4.

Submission: January 3, 2022

Revision received: January 22, 2022 Accepted for publication: January 22, 2022 